\title{
EDITORIAL
}

\section{The link between Cardiovascular Disease and Stroke}

What is good for your heart is also good for your brain.

Coronary artery disease and stroke share common pathophysiology. Coronary heart disease and stroke share many of the same risk factors such as high LDL cholesterol levels, low HDL cholesterol levels, high blood pressure, smoking, diabetes, physical inactivity, and being overweight or obese. Individuals with coronary heart disease, angina, or who have had a heart attack due to atherosclerosis, have more than twice the risk of stroke than those who have not.

Around $20 \%$ of ischaemic stroke are cardiogenic in origin. Cardiac causes can be divided into a) a direct source of embolus or b) a substrate for embolus formation. Direct sources of embolus include left ventricular (LV) apical thrombus in an akinetic LV territory after myocardial infarction (MI), left atrial appendage thrombus in patients with atrial fibrillation (AF), prosthetic valve thrombus in a patient with inadequate anticoagulation, valve vegetations, aortic dissection and cardiac tumours (especially atrial myxoma). Cardiac substrates for embolus formation include patent foraman ovale (PFO), aortic arch atheroma, LV non-compaction, dilated left atrium or dilated cardiomyopathy (DCM).

Common physical finding include $\mathrm{AF}$ is present upto $25 \%$ patients who have a stroke, murmurs involving mitral valve leading to LA enlargement and subsequent $A F$. Uncommonly signs of pulmonary oedema and added heart sounds may be present.

If there is underlying cardiac pathology resulting in thromboembolism, the ECG may show AF, previous MI (anterior $\mathrm{MI}$ in $90 \%$ cases), persistent ST elevation suggesting LV aneurysm \& LVH (hypertension or aortic stenosis). Chest X-Ray may show enlarged heart or features of pulmonary oedema. Contrast echo helps identify LV thrombus. Transoesophageal echo should be performed to assess for aortic arch atheroma, left atrial appendage thrombus and function \& the presence of PFO. For the diagnosis of PFO, the patient is asked to perform the Valsalva manoeuvre and agitated saline is injected peripherally. Upon Valsalva release, immediate passage of bubbles from right atrium to left atrium suggests a PFO.

The aim of treatment is to correct the underlying abnormality $\&$ restoration of sinus rhythm if possible. Percutaneous closure of PFO is recommended in young patients if

a) stroke/TIA where no other cause can be found

b) recurrent embolic events despite medical treatment

c) cerebral embolic events in more than one carotid territory

In some patients who are unable to take anticoagulants a device can be implanted percutaneously in the left atrial appendage (LAA occlusion device). The risk of haemorrhage into an ischaemic stroke if anticoagulated initially outweighs benefits, so patients receive $300 \mathrm{mg}$ aspirin od for 2 weeks before switching to warfarin.

Clinical scenario: If a patient presents with acute stroke followed by acute ST elevation MI a CT head \& aorta is urgently considered. If the scan excludes aortic dissection \& intracranial haemorrhage or large vessel ischemia he should be thrombolysed rather than PPCI as the risk of haemorrhagic transformation or further stroke is high provided the patient presents within the window period of thrombolysis.

\section{Dr. Manzoor Mahmood}

Associate Professor of Cardiology, BSMMU, Dhaka

\section{Prof. Harisul Hoque \\ Professor of Cardiology \& Head, Division of Heart Failure, Preventive Cardiology \& rehabilitation, BSMMU, Dhaka}

\section{Reference:}

1. Medical Masterclass $3^{\text {rd }}$ Edition 2018 published by Royal College of Physicians of London.

2. www.strokeassociation.org accessed online on 23/01/19 